\title{
Adaptação transcultural do Mental States Rating System para o português brasileiro
}

\author{
Cross-cultural adaptation of the Mental States Rating System to Brazilian \\ Portuguese
}

\author{
Patrícia Rivoire Menelli Goldfeld ${ }^{1}$, Daniela Wiethaeuper ${ }^{2}$, Marc-Andrè Bouchard ${ }^{3}$, Luciana \\ Terra $^{4}$, Rosana Baumgardt ${ }^{4}$, Martha Lauermann ${ }^{4}$, Victor Mardini ${ }^{5}$, Claudio Abuchaim ${ }^{6}$, Anne \\ Sordi $^{7}$, Luciana Soares ${ }^{8}$, Lúcia Helena Freitas Ceitlin ${ }^{9}$
}

\begin{abstract}
${ }^{1}$ Psiquiatra. Mestre em Psiquiatria. ${ }^{2}$ Psicóloga. PhD em Psicologia Clínica. Professora adjunta, Universidade do Vale do Rio dos Sinos (UNISINOS), São Leopoldo, RS. ${ }^{3}$ Psychologist. PhD in Clinical Psychology. Full professor, Université de Montréal, Montréal, Québec, Canada. ${ }^{4}$ Psicóloga. ${ }^{5}$ Médico. 6 Psiquiatra. Mestre em Psicologia. ${ }^{7}$ Acadêmica de Medicina, Universidade Federal do Rio Grande do Sul (UFRGS), Porto Alegre, RS. ${ }^{8}$ Acadêmica de Psicologia, UNISINOS. ${ }^{9}$ Psiquiatra. MpH, PhD em Medicina. Professor adjunto, Departamento de Psiquiatria e Medicina Legal, UFRGS.

O presente estudo foi realizado no Programa de Pós-Graduação em Ciências Médicas: Psiquiatria, Departamento de Psiquiatria, Faculdade de Medicina, Universidade Federal do Rio Grande do Sul (UFRGS) e Hospital de Clínicas de Porto Alegre (HCPA), Porto Alegre, RS.
\end{abstract}

\section{Resumo}

Introdução: O artigo apresenta a adaptação transcultural do Mental States Rating System, uma escala de análise de conteúdo do discurso, seja ele falado, descrito ou filmado, que abrange de modo amplo tipos de contratransferência.

Método: Foram realizadas as etapas de equivalência conceitual, equivalência de itens, equivalência semântica, equivalência operacional, equivalência funcional e aprovação da versão final pelo autor original do instrumento.

Resultados: Os critérios de equivalência foram satisfeitos, tendo a versão final sido aprovada pelo autor do instrumento original. Conclusão: A adaptação do Mental States Rating System disponibiliza para uso um instrumento que não só abrange todas as categorias de contratransferência descritas na literatura, mas as amplia, através da Teoria dos Estados Mentais. Constitui, desse modo, uma ferramenta de grande utilidade para pesquisa em psicoterapia e psicanálise, onde a contratransferência tem se mostrado um importante recurso, tornando-se ainda mais crucial nas patologias fundamentadas em estágios precoces do desenvolvimento, nos casos graves e nos traumas severos.

Descritores: Contratransferência, Sistema de Avaliação dos Estados Mentais, adaptação transcultural.

\begin{abstract}
Introduction: This article presents a cross-cultural adaptation of the Mental States Rating System, a content analysis scale applied to spoken, written or taped material, which covers a wide range of countertransference categories.

Method: The following steps were performed: conceptual equivalence, item equivalence, semantic equivalence, operational equivalence, functional equivalence, and approval of the final version by the author of the original instrument.

Results: The study has reached the objectives of equivalence, and the final Brazilian Portuguese version has been approved by the original author.

Conclusion: The study provides a Brazilian Portuguese version of an instrument that not only covers all the range of categories encompassed by countertransference described in the literature, but also expands it through the Mental States Theory. This represents a valuable tool for research on psychotherapy and psychoanalysis, where countertransference has proved to be an important resource, especially for the treatment of diseases based on early stages of development, for severe mental diseases and severe trauma.
\end{abstract}

Keywords: Countertransference, Mental States Rating System, cross-cultural adaptation. 


\section{Introdução}

Uma multiplicidade de fatores converge para a necessidade de se disponibilizar para pesquisa, em português brasileiro, instrumentos de avaliação da contratransferência (CT). A CT necessita ser compreendida e estudada para auxiliar os terapeutas, sendo uma ferramenta indispensável e crucial no entendimento de aspectos inconscientes da dupla paciente/terapeuta ${ }^{1-34}$. Ela ainda é de maior importância no caso de psicopatologias graves ou fundamentadas em estágios precoces do desenvolvimento, onde ocorre prejuízo ou abolição da capacidade de simbolização do indivíduo ${ }^{35-43}$. Além disso, em situações de traumas severos, conforme bem descrito na literatura ${ }^{44-59}$, a capacidade do indivíduo de simbolizar também pode ser perdida ou embotada, sendo seu lugar tomado por uma "equação simbólica" ${ }^{57,60}$. Nesta, a palavra perde significados, carregando o impacto do evento "em si". Em casos ou situações como essas, os sentimentos contratransferenciais permitem aos terapeutas conectar com os elementos inconscientes do paciente, que assim são entendidos para então serem tratados no sentido de produzir alívio do sofrimento.

O termo contratransferência foi utilizado pela primeira vez por Freud em 1909, numa carta a Jung ${ }^{61}$, e depois em 1910, nas "Perspectivas futuras da terapêutica psicanalítica" ${ }^{2}$, para denominar os sentimentos inconscientes do terapeuta que funcionavam como obstáculo ao tratamento - que deveriam ser reconhecidos e sobrepujados ${ }^{63}$. Esse é o conceito clássico de $\mathrm{CT}$. Desenvolvimentos posteriores da teoria e da técnica psicanalítica ampliaram o conceito de modo a servir como instrumento terapêutico e abarcar todos os sentimentos conscientes e inconscientes do terapeuta, o que é denominado conceito totalístico ${ }^{14,15,25,26}$.

Entre os instrumentos para avaliação da CT encontrados na literatura, foi selecionado o Mental States Rating System (MSRS) por tratar-se do instrumento mais completo, que busca avaliar a CT de modo totalístico, abrangendo todas as categorias descritas na literatura sobre o constructo e avaliando-a em seus aspectos conscientes e latentes - para isso, utilizando juízes treinados e com um índice alto de acordo.

O objetivo deste estudo é apresentar o processo de adaptação transcultural do MSRS para o português brasileiro, relativamente à equivalência conceitual, de itens, semântica, operacional, funcional e aprovação da versão final pelo autor.

\section{Mental States Rating System}

A partir de um primeiro instrumento, o Countertransference Rating System ${ }^{64-66}$, Bouchard desenvolveu o MSRS (Bouchard MA, Picard C, Audet C, Brisson SH, Carrier M. Differentiating mental states: therapists, patients, human subjects. Montreal: Université de Montréal; 1998. Scoring manual, unpublished manuscript), que não só abarca o conceito de CT, mas o amplia, através da Teoria dos Estados Mentais. Esta se refere às modalidades ou categorias de atividade intrapsíquica dos indivíduos. No contexto mais específico dessa escala, refere-se às atividades dos terapeutas de orientação analítica ou psicanálise. Assim, dentro do contexto terapêutico, várias formas de atividades psíquicas surgem, sendo algumas com o objetivo de percepção e elaboração de conflitos do mundo interno. O contexto terapêutico também pressupõe capacidade de reconhecimento de estados mentais que seriam influenciados por pulsões e afetos, transformados em formas representáveis e simbólicas, ou seja, mentalização (Lecours \& Bouchard, 1997) ${ }^{67}$.

Trata-se de um instrumento de análise de conteúdo do discurso que apresenta categorias que são prévia e detalhadamente descritas. Aplica-se sobre material escrito ou gravado de sentimentos ou pensamentos de terapeutas logo após sessões ou leitura de vinhetas clínicas. O instrumento serve para a formação de juízes e requer 30 horas de treinamento, no qual exemplos de discurso são cotados individualmente e discutidos em grupo, até que um consenso interjuiz calculado pelo índice kappa de Cohen (Pestana \& Gageiro ${ }^{68}$ atinja um mínimo de 0,70 , considerado excelente como índice de fidelidade (Shrout et al. $)^{69}$. Após, os juízes fazem a cotação do material de pesquisa propriamente dito, individualmente, reunindose apenas para a discussão dos desacordos, para que um consenso seja alcançado e vigore como resultado final.

O MSRS é composto de três grandes categorias: o estado mental objetivo-racional, o reativo pulsional e o reflexivo; e, dentre estas, existem subcategorias (Tabela 1). Cada categoria possui critérios de cotação descritos no manual, assim como exemplos de cotação provindos de outros estudos. Em pesquisas realizadas com esse instrumento, alguns estados mentais têm se mostrado repetidamente antagonistas como o reflexivo e o reativo pulsional (correlação de -0,70), enquanto que o objetivoracional parece não se relacionar com nenhuma das outras duas categorias ${ }^{64,70}$. O acordo interjuiz para a cotação do MSRS variou de 67 a 79\%, com índices kappa variando de 0,63 a 0,87 , indicando um acordo excelente se considerado o efeito do acaso ${ }^{64,70}$.

\section{Método}

Foi utilizado o referencial conceitual proposto por Herdman et al.,72 para adaptação transcultural de instrumentos na área de qualidade de vida, com as sugestões metodológicas propostas por Moraes et al. ${ }^{73}$ 
Tabela 1 - Categorias do Sistema de Avaliação dos Estados Mentais

\begin{tabular}{|c|c|c|}
\hline $\begin{array}{l}\text { Tipo de estado mental } \\
\text { (categoria) }\end{array}$ & Subcategoria & Definição do tipo de estado mental \\
\hline \multirow[t]{4}{*}{ Reativo (REAC) } & DRI & $\begin{array}{l}\text { O indivíduo apresenta sinais de estar em conflito, não } \\
\text { apresenta consciência do fato, e não se consegue identificar } \\
\text { o uso de mecanismos de defesa. }\end{array}$ \\
\hline & $\begin{array}{l}\text { Defensivo primitivo } \\
\text { (LODEF) }\end{array}$ & $\begin{array}{l}\text { O indivíduo apresenta sinais de estar em conflito } \\
\text { inconsciente. Observa-se a presença de afetos mais } \\
\text { primitivos e intensos e o uso de defesas menos evoluídas. }\end{array}$ \\
\hline & $\begin{array}{l}\text { Defensivo de nível } \\
\text { intermediário }\end{array}$ & $\begin{array}{l}\text { O indivíduo apresenta sinais de estar em conflito } \\
\text { inconsciente. Observa-se a presença de afetos comoraiva e } \\
\text { inveja e o uso de defesas como anegação, minimização ou } \\
\text { denegação. }\end{array}$ \\
\hline & $\begin{array}{c}\text { Defensivo avançado } \\
\text { (HIDEF) }\end{array}$ & $\begin{array}{l}\text { O indivíduo apresenta sinais de estar em conflito } \\
\text { inconsciente. Observa-se a presença de afetos como } \\
\text { ansiedade, medo e o uso de defesas mais evoluídas. }\end{array}$ \\
\hline \multirow[t]{3}{*}{ Objetivo-racional (OBR) } & $\begin{array}{l}\text { Objetivo racional per } \\
\text { se (OBR) }\end{array}$ & $\begin{array}{l}\text { O indivíduo assume uma postura de observador distanciado } \\
\text { afetivamente. }\end{array}$ \\
\hline & $\begin{array}{l}\text { Concreto (CONC), } \\
\text { nível psicótico }\end{array}$ & $\begin{array}{l}\text { O indivíduo não mostra um ego orientado para a realidade. } \\
\text { Pensamentos e memórias são experienciados na forma de } \\
\text { vivências sensório-motoras psicóticas, concretas e } \\
\text { imediatas. }\end{array}$ \\
\hline & $\begin{array}{l}\text { Concreto (CONC), } \\
\text { nível não-psicótico }\end{array}$ & $\begin{array}{l}\text { O indivíduo mostra um ego orientado para a realidade. } \\
\text { Pensamentos e memórias são experienciados na forma de } \\
\text { vivências sensório-motoras imediatas, não-psicóticas e } \\
\text { concretas. }\end{array}$ \\
\hline \multirow[t]{3}{*}{ Reflexivo (REF) } & $\begin{array}{l}\text { Reflexivo emergente } \\
\text { (REM) }\end{array}$ & $\begin{array}{l}\text { O indivíduo demonstra alguma capacidade para perceber } \\
\text { uma reação, memória, representação, imagem ou afeto } \\
\text { internos. }\end{array}$ \\
\hline & $\begin{array}{l}\text { Reflexivo empático } \\
\text { (REMP) }\end{array}$ & $\begin{array}{l}\text { O indivíduo manifesta uma capacidade reflexiva adicional, } \\
\text { na forma de empatia básica, que resulta de uma } \\
\text { identificação experimental. }\end{array}$ \\
\hline & $\begin{array}{l}\text { Reflexivo integrativo- } \\
\text { elaborativo (REL) }\end{array}$ & $\begin{array}{l}\text { O indivíduo vai além das formas anteriores de atividade } \\
\text { reflexiva, demonstrando uma maior capacidade de } \\
\text { elaboração do material. Sua situação interna esá } \\
\text { relacionada à situação de um outro significativo em } \\
\text { situações presentes ou passadas. }\end{array}$ \\
\hline
\end{tabular}

na adaptação do instrumento Revised Conflict Tactics Scales, em nosso meio.

A permissão do autor para a tradução e utilização do MSRS foi obtida. Foi montado um grupo de pesquisa formado por dois psiquiatras e um psicólogo com experiência em pesquisa e clínica. Inicialmente, o grupo procedeu à discussão do constructo $\mathrm{CT}$ e estados mentais, buscando compreender os conceitos contidos no instrumento e nos itens. Foi considerado que havia equivalência dos conceitos e itens do instrumento com os conceitos culturais relativos a estados mentais em nosso meio.

A seguir, o instrumento foi traduzido, independentemente, por dois tradutores bilíngües, sendo um deles psiquiatra e um especialista na língua inglesa. Essas duas versões em português foram unificadas por um pesquisador psicanalista, resultando numa terceira versão.

Essa versão foi apresentada a um grupo de cinco profissionais independentes, da área da saúde mental, que discutiu a compreensão e adequação de cada item. $\mathrm{O}$ autor foi contatado para o esclarecimento de dúvidas, quando necessário. A terceira versão em português e as sugestões desse grupo de profissionais foram analisadas quanto à validade de conteúdo por uma dupla de pesquisadores, incluindo um expert no instrumento. Foi, então, elaborada uma quarta versão em português por consenso. Essa última versão foi retrotraduzida por outro 
tradutor independente, bilíngüe, psiquiatra, que não conhecia o instrumento.

$\mathrm{Na}$ etapa seguinte, o grupo de pesquisa procurou analisar o significado referencial de termos/palavras constituintes da escala ${ }^{71}$, comparando a escala original e a versão retrotraduzida, utilizando uma escala analógica visual $^{73}$, e permitindo que a equivalência entre pares fosse pontuada de modo contínuo, de 0 a $100 \%$. Também buscou avaliar o significado geral de cada item, que transcende a literalidade das palavras, levando em consideração o contexto cultural da população-alvo, utilizando-se de uma classificação em quatro níveis: inalterado, pouco alterado, muito alterado ou completamente alterado.

Foram também avaliadas a equivalência operacional - que consiste na possibilidade de a escala ser utilizada na mesma formatação, modo de administração e métodos de mensuração do instrumento original - $\mathrm{e}$ a equivalência funcional, que procura avaliar o quanto o instrumento mede em nosso meio o que se propõe a medir na cultura original. A escala foi utilizada para o treinamento de três juízes, e depois foi medido índice kappa de acordo interjuízes. Para avaliação da equivalência funcional, foi utilizada uma amostragem de 92 terapeutas $^{74}$. Por fim, a escala retrotraduzida foi enviada ao autor original para análise.

\section{Resultados}

A versão final do MSRS está disponível para utilização no Brasil, porém só poderá ser utilizada com a autorização do autor. A discussão do grupo de especialistas a respeito da equivalência conceitual e de itens levou a ajustes lingüísticos e adaptações terminológicas nos termos de 14 itens: em relação à categoria Objetivo-racional, item 2; em relação à categoria Concreto em nível não-psicótico, introdução e itens 1, 3, 4 e 6; em relação à categoria Concreto em nível psicótico, introdução e item 1; em relação à categoria Reflexivo, item 1; em relação às formas da categoria Reflexivo, item 1 e 3; em relação à categoria Defensivo avançado, itens 2 e 3 ; em relação à categoria Defensivo de nível intermediário, itens 2, 3 e 5; e em relação à categoria Defensivo primitivo, itens 2 e 3 .

A avaliação de equivalência semântica (Tabela 2) entre a escala original e a retrotradução evidenciou, de modo geral, uma boa equivalência. Apenas quatro dos 56 itens da retrotradução apresentaram, segundo a escala analógico-visual, um grau de equivalência de significado referencial aquém de $90 \%$ em vista do original. O grupo de pesquisa optou por não mudar esses itens, porque considerou que essas diferenças não alteravam a compreensão dos mesmos. Em relação ao significado geral, observou-se que este se manteve inalterado em $91,3 \%$ dos itens, portanto foi considerado bom.
A avaliação de equivalência operacional mostrou que o instrumento pode ser utilizado na mesma formatação, modo de administração e métodos de mensuração do instrumento original. Para isso, realizouse o treinamento de três juízes no instrumento: um psicólogo e dois psiquiatras. Os juízes receberam um treinamento, realizado por expert no instrumento, de 30 horas de duração, tendo atingido um índice de acordo kappa $=0,81(\mathrm{p}<0,001)$, que é considerado alto. $\mathrm{O}$ instrumento mostrou que apresenta equivalência funcional ao medir em nosso meio o que se propõe a medir na cultura original, o que foi constatado com uma amostra de 92 terapeutas $^{74}$.

$\mathrm{O}$ instrumento retrotraduzido foi enviado ao autor da escala original para análise. $\mathrm{O}$ autor fez poucas observações sobre algumas expressões utilizadas para descrever em inglês alguns estados mentais, indicando a verificação destas na versão em português. Estes comentários foram estudados pelos pesquisadores em conjunto com os tradutores, e foram realizados os ajustes finais na versão em português. $\mathrm{O}$ autor autorizou a utilização da versão final em português.

\section{Discussão}

Herdman et al. ${ }^{72}$ preconizam a utilização de um modo universalista de abordar a pesquisa transcultural, que pretende avaliar e respeitar as diferenças culturais, o que implicaria na necessidade de estabelecer se os conceitos compreendidos em determinado instrumento existem e são interpretados similarmente nas duas culturas em questão; e, se sim, em que grau estes são interpretados similarmente.

A discussão do grupo de experts, para equivalência conceitual e de itens, considerou que os conceitos e itens relativos ao constructo estados mentais utilizados na elaboração do instrumento eram igualmente relevantes em nossa cultura. Além disso, em modo geral, os itens foram considerados adequados na avaliação das três dimensões estudadas: pensamento de tipo reflexivo, defensivo ou objetivo-racional.

O presente estudo realizou uma adaptação transcultural para o português brasileiro de maneira criteriosa, disponibilizando para uso um instrumento muito completo e elaborado para avaliação do constructo estados mentais. $\mathrm{O}$ instrumento ainda necessita ser validado, sendo necessário também medir as suas propriedades psicométricas - confiabilidade, responsividade e validade de constructo (incluindo as propriedades discriminantes, apreciativas e preditivas) - e avaliar o quanto estas são similares ao instrumento original. Parte desse processo de validação já foi realizado, e seus resultados serão apresentados à comunidade científica brevemente ${ }^{74}$. 
Tabela 2 - Processo de análise de equivalência semântica

\begin{tabular}{|c|c|c|}
\hline Itens & A1 VO RT & A2 VO RT \\
\hline $\begin{array}{l}\text { RT Main focus in objective situations and facts. } \\
\text { V3 Foco predominante em situações e fatos objetivos. } \\
\text { T1 Foco predominante em situações e fatos objetivos. } \\
\text { T2 Foco predominante sobrefatos e situações objetivos. } \\
\text { VO Predominant focus on objective facts and situations. }\end{array}$ & $100 \%$ & IN \\
\hline $\begin{array}{l}\text { RT A reality-oriented ego with a logical grammatical structure available is } \\
\text { observed. } \\
\text { V3 Observa-se um ego orientado para a realidade, tendo disponível uma } \\
\text { estrutura gramatical lógica. } \\
\text { T1 Uma disposição egóica orientada para a realidade e uma estrutura } \\
\text { gramatical lógica são observadas. } \\
\text { T2 Há um ego orientado à realidadeque apresenta uma estrutura lógico- } \\
\text { gramatical. } \\
\text { VO A reality-oriented ego displaying a logico-grammatical structure is } \\
\text { available. }\end{array}$ & $91 \%$ & IN \\
\hline $\begin{array}{l}\text { RT Some safety seeking process is clearly activated, but the subject does not } \\
\text { seem to be aware of the fact. } \\
\text { V3 Algum processo de busca de segurança está claramente ativado, mas o } \\
\text { sujeito não se mostra consciente do fato. } \\
\text { T1 Algum processo de busca de segurança está claramente ativado, mas o } \\
\text { sujeito não se mostra consciente do fato. } \\
\text { T2 Algum processo claramente identificável de busca de segurança está } \\
\text { ativado e permanece fora da consciência imediata do sujeito. } \\
\text { VO Some clearly identifiable security seeking process is activated that is and } \\
\text { remains outside of the subject's immediate awareness. }\end{array}$ & $95 \%$ & IN \\
\hline $\begin{array}{l}\text { RT The subject demonstrates some listening or opening to some internal } \\
\text { reaction, memory, representation, image, or affect. } \\
\text { V3 O sujeito demonstra alguma escuta ou abertura a alguma reação interna, } \\
\text { memória, representação, imagem ou afeto. } \\
\text { T1 O sujeito demonstra alguma escuta ou abertura a alguma reação, memória, } \\
\text { representação, imagem ou afeto internos. } \\
\text { T2 O sujeito demonstra ouvir um pouco ou uma certa abertura a uma reação, } \\
\text { lembrança, representação, imagem, afeto interno. } \\
\text { VO The subject demonstrates some listening or openness to an inner } \\
\text { reaction, memory, representation, image, affect. }\end{array}$ & $100 \%$ & IN \\
\hline
\end{tabular}

A1 = pontuação de equivalência referencial; A2 = pontuação de equivalência geral; IN = inalterado; RT = versão retrotraduzida; $\mathrm{T} 1$ = tradutor $1 ; \mathrm{T} 2=$ tradutor $2 ; \mathrm{V} 3=$ versão unificada por experts; $\mathrm{VO}=$ versão original.

\section{Referências}

1. Balint A, Balint M. Original papers on transference and countertransference. Int J Psychoanal. 1939;3-4:223-30.

2. Baranger M. A mente do analista: da escuta à interpretação. Rev Bras Psic. 1992;26(4):573-86.

3. Baranger W, Baranger M. La situación analítica como campo dinâmico. In: Problemas del campo psicoanalítico. Buenos Aires: Kargieman; 1961. p. 129-64.

4. Bion WR. On arrogance. Int J Psychoanal. 1958;39(2-4):144-6.

5. Etchegoyen RH. Contratransferência. In: Etchegoyen RH. Fundamentos da técnica psicanalítica. Porto Alegre: Artmed; 1987. p. 143-65.

6. Favali PH. Campo e intersubjetividade. In: Eizirik CL, Aguiar R, Schestatsky SS, editors. Psicoterapia de orientação analíticafundamentos teóricos e clínicos. Porto Alegre: Artmed; 2005. p. 141-56.

7. Feldman M. Projective identification: the analyst's involvement. Int J Psychoanal. 1997;78(Pt 2):227-41.

8. Fenichel O. [Problems of psychoanalytic technique]. Rev Fr Psychanal.
1951;15(4):506-26; concl.

9. Ferenczi S, Rank O. The development of psycho-analysis. New York: Dover; 1923. p. 28-44.

10. Ferenczi S. On the technique of psycho-analisys. In: Jones E, editor. Further contributions to the theory and technique of psycho-analisys. London: International Psycho-Analytical Library; 1951. p. 177-89.

11. Gabbard GO. Countertransference issues in psychiatric treatment. In: Oldham JM, Riba MB, editors. Review of psychiatry (Series). Washington: American Psychiatry; 1999.

12. Grinberg L. Projective counteridentification and countertransference. In: Epstein L, Feiner A, editors. Countertransference. New York: Jason Aronson; 1977. p. 169-91.

13. Hafkenscheid A. Objective countertransference: do the patients' interpersonal impacts generalize across therapists? Clin Psychol Psychother. 2003;10(1):31-40.

14. Heimann P. Acerca de la contratransferência. Rev Uruguaya Psicoanal. 1961-62;iv(1):129-35.

15. Heimann P. Counter-transference. II. Br J Med Psychol. 1960;33:9-15.

16. Holmqvist R, Armelius BA. The patient's contribution to the therapist's countertransference feelings. J Nerv Ment Dis. 1996;184(11):660-6. 
17. Jacobs TJ. Countertransference past and present. In: Michels R, Abensour L, Eizirik CL, Rusbridger R, editors. Key papers on countertransference. London: Karnak; 2002.

18. Kyrle M. Contratransferência normal e alguns de seus desvios. In: Money-Kyrle R. Obra selecionada. São Paulo: Casa do Psicólogo; 1996.

19. Lacan J. A ética da psicanálise. In: Lacan J. O seminário. Vol 7. Rio de Janeiro: Jorge Zahar; 1995. p. 349-61.

20. Lindy JD, Wilson JP. Empathic strain and countertransference roles: case illustrations. In: Wilson JP, Lindy JD. Countertransference and the treatment of PTSD. New York: Guilford; 1994. p. 62-85.

21. Metcalf LM. Countertransference among play therapists: implications for therapist development and supervision. Int J Play Ther. 2003;12(2):31-48

22. Ogden TH. On projective identification. Int J Psychoanal. 1979;60:35773.

23. Ogden TH. Projective identification and psychotherapeutic technique. New York: Janson Aronson; 1982.

24. Ogden TH. Subjects of analysis. Northvale: Janson Aronson; 1994.

25. Racker H. Estudos sobre técnica psicanalítica. $2^{\mathrm{a}}$ ed. Porto Alegre: Artmed; 1986.

26. Racker H. The meanings and uses of countertransference. Psychoanal Quarterly. 1957;26:303-57.

27. Reik T. New ways in psychoanalytic technique. Intern J Psychoanal; 1933;14:321-34

28. Reik T. The surprised psycho-analyst. In: Farrar, Straus, editors Listening with the third ear. New York: Farrar, Straus; 1948. p. 23.

29. Rusbridger R. Introduction. In: Michels R, Abensour L, Eizirik CL, Rusbridger R. Key papers on countertransference. London: Karnak; 2002.

30. Segal H. Countertransference. In: Alexandris A, Vastamatzis G. Countertransference: theory, technique, teaching. London: Karnak; 1993. p. 13-20.

31. Thöma H, Kachele H. Contratransferência. In: Thöma H, Kachele H. Teoria e prática da psicanálise. Porto Alegre: Artmed; 1992. p. 96-113.

32. Winnicott DW. Hate in countertransference. Intern J Psychoanal. 1949;2:69-74.

33. Wolstein B. Essential papers on countertransference. New York: University; 1988.

34. Zimerman DE. Contratransferência. In: Fundamentos psicanalíticos. Porto Alegre: Artmed; 1999. p. 347-67.

35. Kernberg O. Borderline personality organization. J Am Psychoanal Assoc. 1967;15:641-85.

36. Kernberg O. Severe personality disorders. New York: Yale University; 1984.

37. McDougall J. Primitive communication and the use of countertransference. In: Epstein L, Feiner A, editors. Countertransference. New York: Jason Aronson; 1979;267-303.

38. McIntyre SM, Schwartz RC. Therapists' differencial countertransference reactions toward clients with major depression or borderline personality disorder. J Clin Psychology. 1998;54(7):92331.

39. Rosenfeld HA. Notes on the psychoanalysis of the superego conflict of an acute schizophrenic patient. Intern J Psychoanal. 1952;31:111-31.

40. Bion WR. Differentiation of the psychotic from the non-psychotic personalities. Intern J Psychoanal. 1957;38:266-75.

41. Bion WR. Language and the schizofrenic. In: Klein M, Heimann P, Money-Kyrle RE, editors. New directions in psychoanalysis. London: Tavistok; 1955. p. 220-39.

42. Bion WR. Attacks on linking. Intern J Psychoanal. 1959;40:311-5.

43. Bollas C. The shadow of the object. psychoanalysis of the unthought known. New York: Columbia University; 1987.

44. Armsworth MW. Therapy of incest survivors: abuse or support? Child Abuse and Neglect; 1989;13:549-62.

45. Burnstein A. Treatment noncompliance in patients with post-traumatic stress disorder. Psychosomatics. 1986;27(1):37-40

46. Butterfield MI, Panzer PG, Forneris CA. Victimization of women and its impact on assessment and treatment in the psychiatric emergency setting. Psychiatric Clin North Am. 1999;22(4):875-96.

47. Colao FE, Hunt M. Therapists coping with sexual assault. In: Robbins JH, Siegel RJ, editors. Women changing therapy: new strategies in feminist therapy. New York: Haworth; 1983. p. 205-14.
48. Dutton MA, Rubinstein F. Working with people with PTSD. 1995.

49. Faber B. Stress and burnout in the human service professions. New York: Pergamon; 1983.

50. Figley CR. Epilogue: the transmission of trauma. In: Compassion fatigue: coping with secondary traumatic stress disorder in those who treat the traumatized. New York: Brunner/Mazel; 1995.

51. Fox R, Carey LA. Therapist's collusion with the resistance of rape survivors. Clin Soc Work J. 1999;27(2):185-201.

52. Herman JL. Trauma and recovery. New York: Basic Books; 1992.

53. Jenkins SRE, Baird S. Secondary traumatic stress and vicarious trauma: a validational study. J Traum Stress. 2002;15(5):423-32.

54. Krupnick J. Brief psychotherapy with victims of violent crime. Victimology: An Intern J. 1980;5(2-4):347-54.

55. Maslach C, Jackson SE. The measurement of experienced burnout. J Occup Behav. 1981;2.

56. McCann IL, Pearlman LA. Through a glass darkly: understanding and treating the adult trauma survivor through constructivist self development theory. New York: Brunner/Mazel; 1990.

57. McCann IL, Sakheim D, Abrahamson D. Trauma and victimization: a model of psychological adaptation. Couns Psychol. 1988;16(4):53194.

58. Mitrani J. Toward an understanding of unmentalised experience. Psychoanal Quarterly. 1995;64:68-112.

59. Pearlman LA, Saakvitne KW. Trauma and the therapist: countertransference and vicarious traumatization in psychotherapy with incest survivors. New York: WW Norton; 1995. p. 279-94, 359-66.

60. McDougall J. Primitive communication and the use of countertransference. In: Epstein L, Feiner A, editors. Countertransference. New York: Jason Aronson; 1979. p. 267-303.

61. Freud S, Jung CG. The Freud/Jung Letters. Princetown: Princetown University; 1974.

62. Freud S. As perspectivas futuras da terapêutica psicanalítica. In: Edição standard brasileira das obras completas de Sigmund Freud. vol. XI. Rio de Janeiro: Imago; 1969. p. 125-36.

63. Freud S. Observações sobre o amor transferencial. In: Edição standard brasileira das obras completas de Sigmund Freud. vol. XII. Rio de Janeiro: Imago; 1969. p. 208-22.

64. Lecours S, Bouchard MA, Normandin L. Countertransference as the therapist's mental activity: experience and gender differences among psychoanalytically oriented psychologists. Psychoanal Psychology. 1995; 12:59-279.

65. Normandin L, Bouchard MA. The effects of theoretical orientation and experience on rational, reactive and reflective countertransference. Psychoter Res. 1993;3(2):77-94.

66. Normandin L, Bouchard MA. Vers une vision integrée et opérationnelle du contre-transfert: présentation d'une grille d'analyse. Rev Can Sci Comportement. 1991.

67. Lecours S, Bouchard MA. Dimensions of mentalisation: outlining levels of psychic transformation. Int J Psychoanal. 1997;78(Pt 5):85575 .

68. Pestana MH, Gageiro JN. Análise de dados para ciências sociais. $2^{\mathrm{a}}$ ed. Lisboa: Sílabo; 2000

69. Shrout PE, Spitzer RL, Fleiss JL. Quantification in psychiatric diagnosis revisited. Arch Gen Psychiatry. 1987;44(2):172-7.

70. Lecours S, Bouchard MA, Normandin L. Countertransference as the therapist's mental activity: experience and gender differences among psychoanalytically oriented psychologists. Psychoanal Psychol. 1995; $12: 259-79$.

71. Seguin MH, Bouchard MA. Adaptive regression and countertransference mental activity. Psychoanal Psychol. 1996;13(4):457-74.

72. Herdman M, Fox-Rushby J, Badia X. A model of equivalence in the cultural adaptation of HRQoL instruments: the universalist approach. Qual Life Res. 1998;7(4):323-35.

73. Moraes CL, Hasselmann MH, Reichenheim ME. Adaptação transcultural para o português do instrumento "Revised Conflict Tactics Scales (CTS2)" utilizado para identificar violência entre casais. Cad Saude Publica. 2002;18(1):163-76.

74. Goldfeld P, Terra L, Abuchaim C, Sordi A, Wiethaeuper D, Bouchard MA, et al. Mental states as part of countertransference responses in psychotherapists facing reports of traumatic events of mourning and sexual violence. Psychother Res. 2008. In Press. 